\title{
An Optically Controlled Module for Wavelength Conversion Circuits
}

\author{
Georgios I. Papadimitriou \\ Department of Informatics, \\ Aristotle University of \\ Thessaloniki, P.O. Box \\ 888, 54124 Thessaloniki, \\ Greece \\ gp@,csd.auth.gr
}

\author{
Amalia N. Miliou \\ Department of Informatics, \\ Aristotle University of \\ Thessaloniki, P.O. Box \\ 888, 54124 Thessaloniki, \\ Greece \\ amiliou@csd.auth.gr
}

\author{
Andreas S. Pomportsis \\ Department of Informatics, \\ Aristotle University of \\ Thessaloniki, P.O. Box \\ 888, 54124 Thessaloniki, \\ Greece \\ apombo@csd.auth.gr
}

\begin{abstract}
We report an innovative solely optical architecture to implement the centralized wavelength conversion module of the CWC (Controlled Wavelength Conversion) protocol [1]. The proposed module was designed to distribute the conversion wavelengths to the corresponding converters in an unbiased manner taking into consideration the information carried by the control wavelength. The design of the Optically Controlled Module is based on the use of optical logic circuits. These optical circuits distribute the available wavelengths by eliminating the need of optical to electronic translation of the network's feedback information and thus reducing drastically processing time. Furthermore, due to the all-optical nature of the network hub the reliability of the system is improved.
\end{abstract}

\section{Introduction}

The design of emerging single-hop lightwave local and metropolitan area networks [2] is based on Wavelength Division Multiplexing (WDM) in order to achieve Gigabit data rates.

Due to the limited speed of the stations' electronic circuits, single channel optical networks - such as FDDI, DQDB, Fasnet, Expressnet, etc - have not proved capable of supporting Gigabit data rates. The WDM technique [2] circumvents this limitation by dividing the available optical bandwidth into multiple channels of lower bandwidth, which can be easily supported by the stations' electronic circuits. Furthermore, multiplexing and demultiplexing of the multiple channels are performed in the optical domain without the need of optical to electronic translation and vice versa. As a result, the WDM technique allows the implementation of all-optical networks, which are capable of providing Gigabit data rates by using present-day optical and electronic technology.
Broadcast-and-Select Star networks, a specific category of WDM networks, use a Passive Star Coupler to broadcast all inputs to all outputs. However, networks of this architectural form suffer from low efficiency when the offered traffic is bursty. Under bursty traffic conditions, it is likely that some wavelengths are idle while some other get overloaded. As a result, the overall network performance is compromised and the system degraded.

The recently introduced MAC protocol is capable of operating efficiently under bursty traffic conditions. According to the CWC protocol [1] an array of tunable wavelength converters is placed at the network hub so that the incoming packets are uniformly distributed at the available wavelengths. This way, the load is balanced between the wavelengths and consequently, the network performance is improved. However, the problem of optical to electronic translation at the controller still remains.

In this paper, a solely optical architecture implementing the centralized wavelength conversion module of the CWC protocol is introduced as a novel way to further enhance network performance. The proposed architecture is simulated by means of the XHATCH simulation package [7] and it is proved that it successfully implements the functionality required by the CWC protocol.

The paper is organized as follows: The CWC protocol is briefly described in section 2 . The proposed solely optical centralized control module architecture is presented in Section 3. Section 4 presents the results of the simulation and the functionality of the proposed architecture is confirmed using the XHATCH simulation package. Finally, concluding remarks are given in Section 5.

\section{The CW Architecture}

A network using the CWC protocol can be analyzed into two basic modules: The Broadcast-and- 
Select Module and the Centralized Wavelength Conversion Module.

A set of stations is defined as $U=\left\{\mathrm{u}_{1}, \ldots, \mathrm{u}_{\mathrm{N}}\right\}$, where $\mathrm{N}$ is the number of stations. To simplify the description of the network, we assume that all stations have equal distance from the network hub. Each station is placed $\mathrm{D} / 2$ slots away from the hub so that the round trip propagation delay from all stations to the hub is equal to D slots.

A set of W wavelengths, defined as $\Lambda=\left\{\lambda_{1}, \ldots, \lambda_{\mathrm{W}}\right\}$, are used for data transmission (data wavelengths), while another wavelength $\lambda_{c}$, common for all stations, is used for the transmission of control signals (control wavelength). The time axis in data wavelength is divided into data time slots, each being equal to the packet transmission time. The control wavelength is addressed in a TDM manner. In the control wavelength the time axis is divided into control slots and each control slot is divided into $\mathrm{N}$ minislots. Each minislot has a length of $\left[\log _{2} \mathrm{~N}\right]$ bits and corresponds to a specific source node. In order to preserve synchronization, control and data slots are of equal length.

Each source node $u_{k}(k=1, \ldots, N)$ is set with two fixed-wavelength lasers: one at a data wavelength $\lambda_{\text {ik }}$ $\left(\mathrm{i}_{\mathrm{k}}=[\mathrm{kW} / \mathrm{N}] ; 1 \leq \mathrm{i}_{\mathrm{k}} \leq \mathrm{W}\right)$ and one at the control wavelength $\lambda_{\mathrm{c}}$. In order to simplify the protocol presentation, it is assumed that $\mathrm{N} / \mathrm{W}$ is an integer. Thus, each data wavelength is shared by N/W source nodes. Furthermore, the data and the control wavelength are combined by means of a $2 \times 1$ combiner.

As shown in Figure 1, the optical signal is fed to a Passive Star Coupler after passing through a wavelength conversion mechanism. At each receiver, the optical signal is split into two parts by means of an $1 \times 2$ splitter. One part of the signal goes to a fixed optical filter passing only the control wavelength and the other part is fed to a tunable optical filter that can be tuned to allow passage to any of the $\mathrm{W}$ data wavelengths.

Whenever a source node has a packet to transmit, it sends a message containing the source and the destination node of the packet through the control wavelength and the packet is transmitted in the next time slot. Each destination node continuously monitors the control wavelength by analyzing the control information. When it locates a data packet to arrive in the next time slot, it tunes its optical filter to the corresponding wavelength and receives the incoming packet.

The random transmission strategy was chosen, instead of FIFO transmission strategy, because it achieves a significantly higher performance [1]. Packet transmission is based on pipelining. Thus, a ready source node continuously transmits packets without waiting for an acknowledgement. All the transmitted but unacknowledged packets are kept in a buffer.

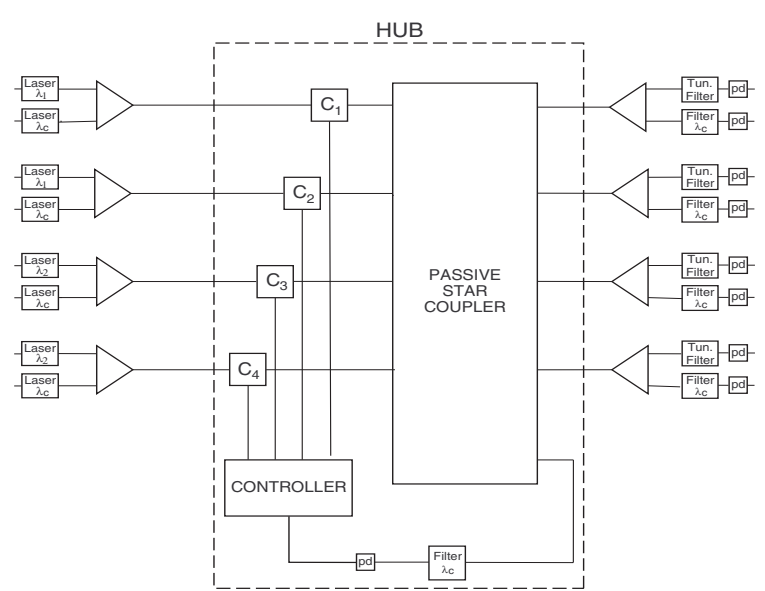

Fig. 1: A WDM star network operating under the CWC protocol (Four stations).

The centralized wavelength conversion module is an array of electronically tunable wavelength converters, $C_{k}(k=1, \ldots, N)$, which are capable of converting the wavelengths of the transmitted packets while preserving the control wavelength $\lambda_{c}$. At any time slot t, each wavelength converter $C_{k}$ can be tuned to convert the fixed incoming wavelength $\lambda_{\mathrm{ik}}$ to any wavelength $\lambda_{\mathrm{jk}} \in \Lambda$. The state of the wavelength converters $C_{k}(k=1, \ldots, N)$ is determined by a controller according to a Wavelength Conversion Algorithm [1].

\section{The Optical Implementation of the Control Module}

In this section, we introduce a solely optical architecture implementing the centralized wavelength conversion module of the CWC protocol. The optically wavelength conversion module is designed to distribute 


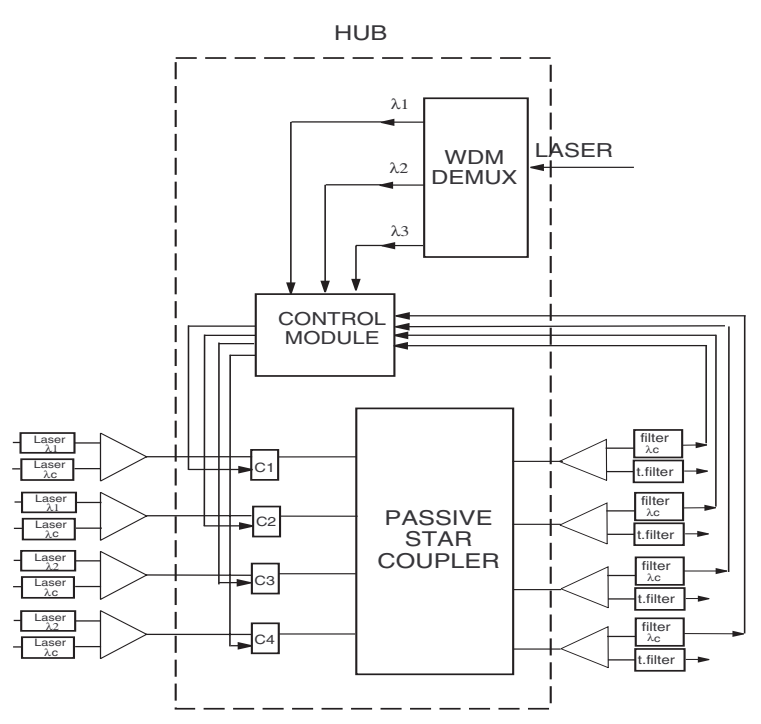

Fig. 2: A WDM star network with the optical wavelength conversion module.

the $M$ conversion wavelengths $\left\{\lambda_{1}, \ldots, \lambda_{\mathrm{m}}\right\}$ to the corresponding $\mathrm{N}$ converters in an unbiased manner taking into consideration the information carried by the control wavelength $\left(\lambda_{c}\right)$. The conversion wavelengths are provided by a constant laser source.

The $\lambda_{c}$ is carrying information from each source node regarding an imminent transmission of a packet in the next time slot and it has a value of ' 1 ' when a transmission is expected or ' 0 ' otherwise. This information has to be transferred back to the control module in order to redistribute the conversion wavelengths for the next round.

Figure 2 shows an example of the network that operates under the CWC protocol. The illustrated example uses four stations, two data wavelengths $\left(\lambda_{1}\right.$ and $\lambda_{2}$ ), and a constant laser source, which provides three conversion wavelengths $\left(\lambda_{1}, \lambda_{2}\right.$, and $\left.\lambda_{3}\right)$.

The core of the optical wavelength conversion module is the control module. The control module is an array of M Optical Logic Circuits (OLCs) (Figure 3). The OLC is an optical device with $\mathrm{N}$ input and $\mathrm{N}$ output ports. At each time slot, only one of the incoming signals is allowed to pass to the corresponding output port [3] while the others are blocked. The selection of the incoming signal, which is allowed to pass, is based on the value of a laser clock pulse. Each OLC is associated with one of $\mathrm{M}$ conversion wavelengths.

$\mathrm{N}$ AND gates were utilized with each OLC. The input signals to the N AND gates are the combined $\mathrm{N}$ $\lambda_{\mathrm{c}} \mathrm{s}$ of the outputs of the Star Coupler and the associated conversion wavelengths and the output of the N AND gates are the input signals of the OLCs.

Moreover, 2N AND-NOT and N AND gates were implemented at the output of the $1 \mathrm{xN} \lambda_{\mathrm{c}}$ splitters. These gates were employed in order to prevent reuse of the corresponding outputs of the subsequent OLCs inactivating those outputs from which a signal had emerged, Figure 3.

In our example, three OLCs were utilized with four inputs and four outputs each and three different conversion wavelengths $\lambda_{i}(i=1,2,3)$. Moreover, for inactivating the already used OLC outputs, eight ANDNOT gates and four AND gates were used, i.e. when a signal emerges from the first output port of OLC1 then the first output ports of OLC2 and OLC3 are inactive (Figure 3).

\subsection{Directional Coupler Implementation}

In our design, we consider the system to be all optical because all signals are in optical form with the exception of a high speed electronic signal used to control the switches, gates, etc [5].

The well-developed technology of $\mathrm{LiNbO}_{3}$ directional coupler was chosen to implement the logic elements of the system [4] due to its low crosstalk and low insertion loss, as well as the simplicity of the device.

The key element in the design is a switch with two optical inputs A and B and two optical outputs D and E while there is also an electronic input $\mathrm{C}$. In the absence of input signal at terminal $\mathrm{C}$, the device is working at the cross state, whereas, the presence of input signal at terminal $\mathrm{C}$ will cause the device to work at the bar state.

For the low level design of the system, we used an AND-NOT gate and an AND gate corresponding to cross and bar state of the switch, respectively while an AND-NOT gate in conjunction with a wired OR gate is acting as a combiner. A single mode optical fiber was used for interconnections and as a delay element.

Our main concern using a $\mathrm{LiNbO}_{3}$ switch in the circuit was the large physical size and its sensitivity to polarization. The first issue was not a limiting factor in the actual size of the network since CWC is placed centrally in the hub of the network. Polarization was addressed by using in-line polarization controllers at all inputs. 


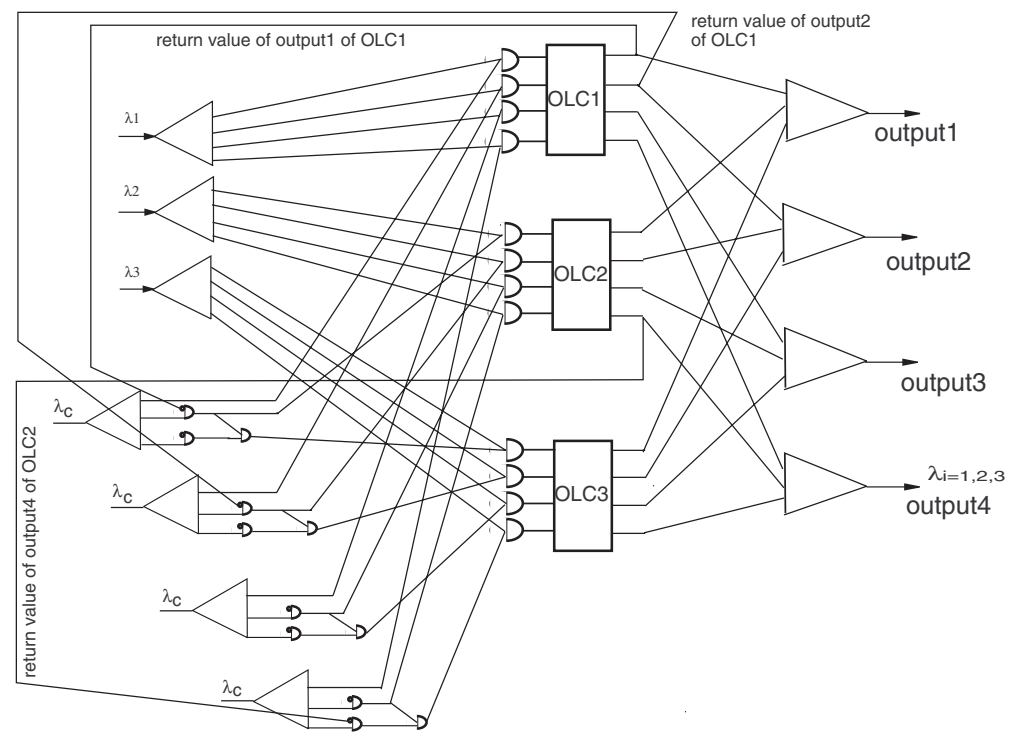

Fig. 3: The control module.

A laser diode allows for either a $\sim 1300 \mathrm{~nm}$ or $\sim 1500 \mathrm{~nm}$ source signal with a pulse power adjusted to $1 \mathrm{~mW}$. Moreover, the splitters and the combiners in the circuit are exhibiting $3 \mathrm{~dB}$ insertion loss, fixed directional couplers. Losses for the switch are determined experimentally. Specifically, the insertion loss of the switch may range up to $5 \mathrm{~dB}$, while the crosstalk is less than $-20 \mathrm{~dB}$ [5]. These loss figures are the controlling parameters for the optical power required in the circuit and they affect the number of stages the signal can travel before there is a need for amplification. In our design with $1 \mathrm{~mW}$ of pulse power, the signal traveling through three switches is attenuated by almost $97 \%$. For this reason, we propose the use of line amplifiers at places along the path of the signal to counteract the losses incurred. To maintain the all-optical nature of the system, we suggest the use of erbium doped fiber amplifiers [6]. In addition, by using a dichroic coupler the signal and the optical pumping are inserted into the piece of $\mathrm{Er}^{3+}$-doped fiber. This type of amplifier limits the laser diode output to $\sim 1500 \mathrm{~nm}$ and the overall design allows up to $22 \mathrm{~dB}$ gain over $35 \mathrm{~nm}$ bandwidth.

\section{XHATCH Simulation and Results}

To test the functionality of the control module (Figure 3), the XHATCH CAD simulator [7] was used and its operation was verified (Figures 4-6). The output for all combinations of information received by $\lambda_{c} s$ is presented in Table 1.

Table 1: Output results for all combinations of information received by $\lambda_{c} s$.

\begin{tabular}{|c|c|c|c|c|}
\hline \multirow[t]{2}{*}{ Input } & \multicolumn{4}{|c|}{ Output } \\
\hline & 1 & 2 & 3 & 4 \\
\hline 1111 & $\lambda 1$ & $\lambda 2$ & $\lambda 3$ & - \\
\hline 1110 & $\lambda 1$ & $\lambda 2$ & $\lambda 3$ & - \\
\hline 1101 & $\lambda 1$ & $\lambda 2$ & - & $\lambda 3$ \\
\hline 1100 & $\lambda 1$ & $\lambda 2$ & - & - \\
\hline 1011 & $\lambda 1$ & - & $\lambda 2$ & $\lambda 3$ \\
\hline 1010 & $\lambda 1$ & - & $\lambda 2$ & - \\
\hline 1001 & $\lambda 1$ & - & - & $\lambda 2$ \\
\hline 1000 & $\lambda 1$ & - & - & - \\
\hline 0111 & - & $\lambda 1$ & $\lambda 2$ & $\lambda 3$ \\
\hline 0110 & - & $\lambda 1$ & $\lambda 2$ & - \\
\hline 0101 & - & $\lambda 1$ & - & $\lambda 2$ \\
\hline 0100 & - & $\lambda 1$ & - & - \\
\hline 0011 & - & - & $\lambda 1$ & $\lambda 2$ \\
\hline 0010 & - & - & $\lambda 1$ & - \\
\hline 0001 & - & - & - & $\lambda 1$ \\
\hline 0000 & - & - & - & - \\
\hline
\end{tabular}

The wavelengths shown at Table 1 will be the corresponding conversion wavelengths used by $C_{k}$ $(\mathrm{k}=1, \ldots, 4)$. The occurrence probability of each input of Table 1 depends on the network load. For example, if 
the network is heavily loaded, inputs with a large number of $1 \mathrm{~s}$ have much higher occurrence probabilities than inputs with a small number of $1 \mathrm{~s}$. The opposite happens when the network is lightly loaded. Therefore, the expected network load should be taken into account in order to decide how many conversion wavelengths are required for the implementation of the specific network.

To verify further the functionality of our design Table 2 shows, for the above highlighted combination, the evolution of the output in the next four time slots.
Table 2: Output's evolution for $\lambda_{\mathrm{c}} \mathrm{s}=\left(\begin{array}{llll}1 & 1 & 0 & 1\end{array}\right)$.

\begin{tabular}{|l|l|l|l|l|l|}
\hline Input & Output & $\mathrm{t} 4$ & $\mathrm{t} 3$ & $\mathrm{t} 2$ & $\mathrm{t} 1$ \\
\hline \multirow{3}{*}{1101} & 1 & $\lambda 2$ & $\lambda 2$ & $\lambda 3$ & $\lambda 1$ \\
\cline { 2 - 6 } & 2 & $\lambda 3$ & $\lambda 3$ & $\lambda 1$ & $\lambda 2$ \\
\cline { 2 - 6 } & 3 & - & - & - & - \\
\cline { 2 - 6 } & 4 & $\lambda 1$ & $\lambda 1$ & $\lambda 2$ & $\lambda 3$ \\
\hline
\end{tabular}

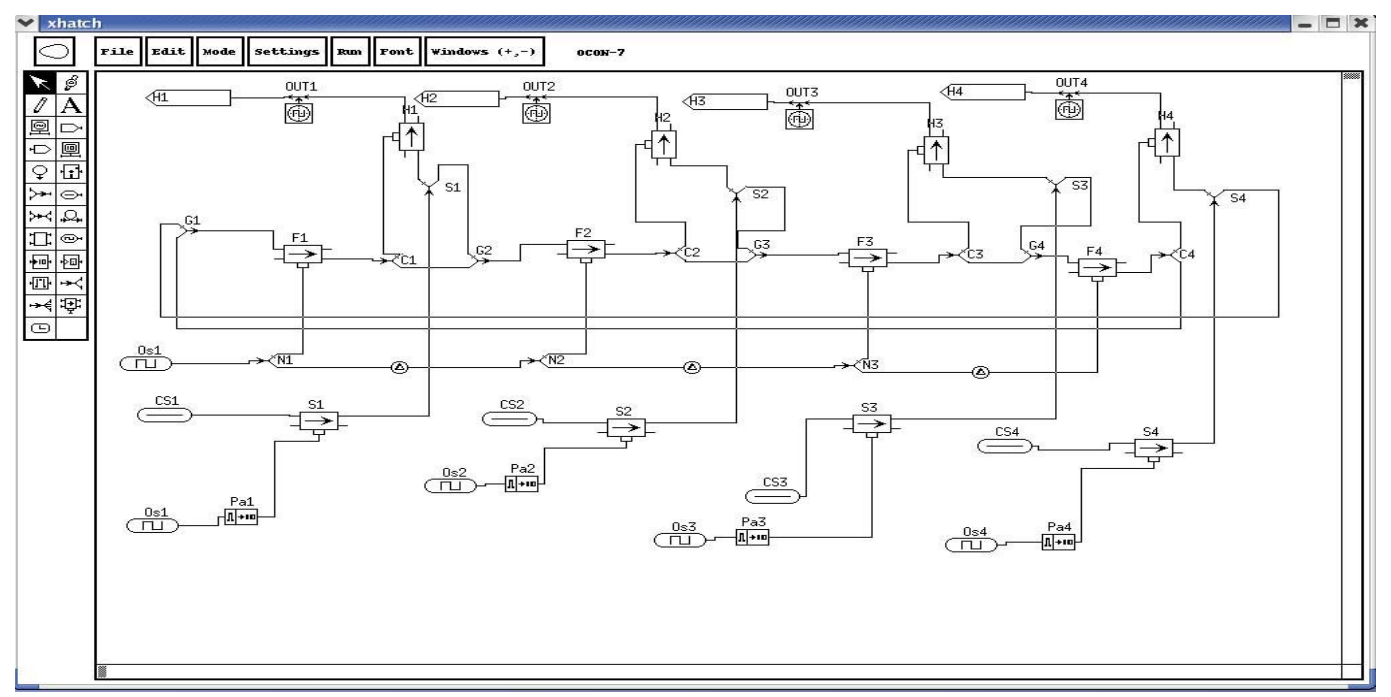

Fig. 4: The XHATCH layout of the first optical logic circuit (OLC1).

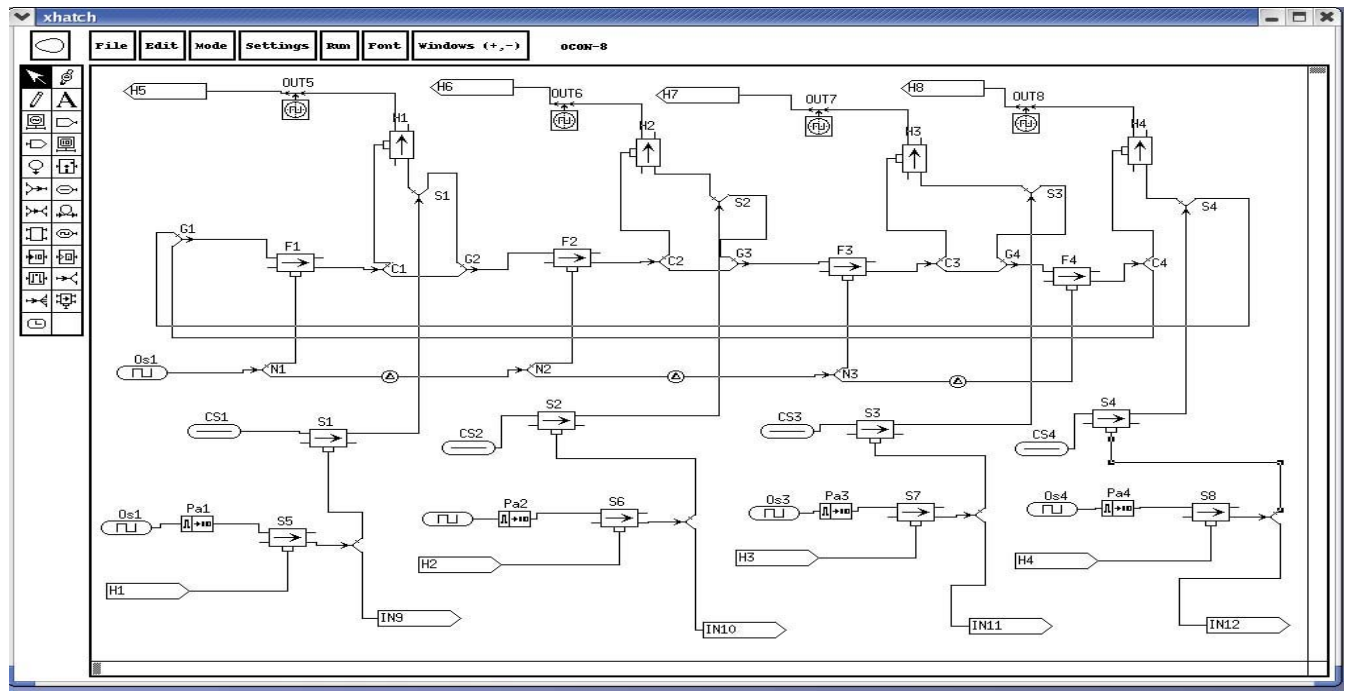

Fig. 5: The XHATCH layout of the second optical logic circuit (OLC2). 


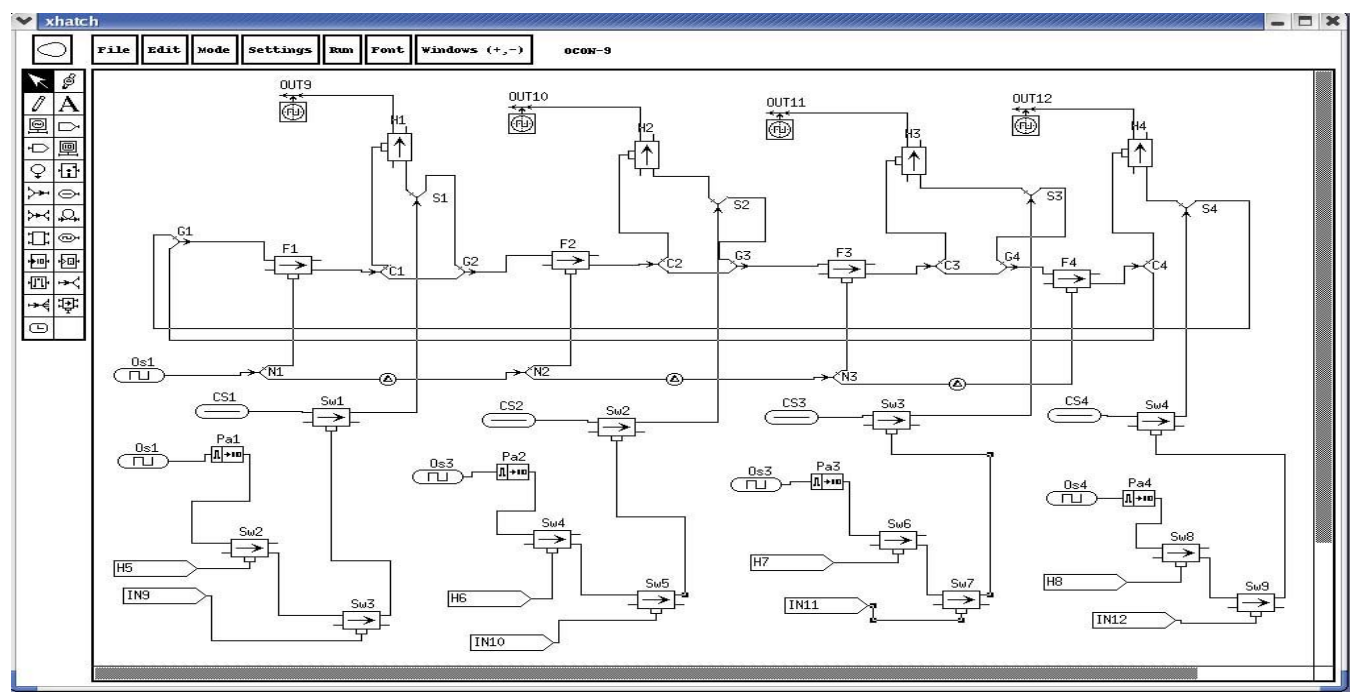

Fig. 6: The XHATCH layout of the third optical logic circuit (OLC3).

Figure 7 shows the results of Table 2 using the $\mathrm{XHATCH}$ simulator. The repetition of wavelength distribution at $\mathrm{t} 3$ and $\mathrm{t} 4$ is caused by the disabled input and the property of OLC to shift cyclically the allowed output ports (widening of the pulse in Figure 7).

It is evident from Figure 7 that at the first time slot, $\lambda_{1}$ will be the output of OLC1 from port 1, inactivating port1 from OLC2 and OLC3 and this is going to be the conversion wavelength used by $\mathrm{C}_{1}$. Moreover, $\lambda_{2}$ will be the output of OLC2 from port 2 (it is denoted as OUT6 in Figure 7) inactivating output 2 of OLC3, while $\lambda_{3}$ will be the output of OLC3 from port 4 (denoted as OUT12). The $\lambda_{2}$ and $\lambda_{3}$ will be the conversion wavelengths used by $\mathrm{C}_{2}$ and $\mathrm{C}_{4}$ correspondingly.

At the second time slot, OLCs will shift cyclically the allowed output ports. Therefore, $\lambda_{1}$ will be the output of OLC1 from port 2, inactivating port2 from OLC2 and OLC3, $\lambda_{2}$ will be the output of OLC2 from port 4 (denoted as OUT8), inactivating port 4 from OLC3, and $\lambda_{3}$ will be the output of OLC3 from port 1 (denoted as OUT9). This procedure will continue at the next time slots providing a fair distribution of the conversion wavelengths to the wavelength converters.

The proposed circuit is working with different combinations of input-output ports and with different number of wavelengths. However, in WDM broadcastand-select star networks each wavelength is shared by more than one source nodes. Moreover, a large number of input-output ports would lead to an increase of the network complexity.

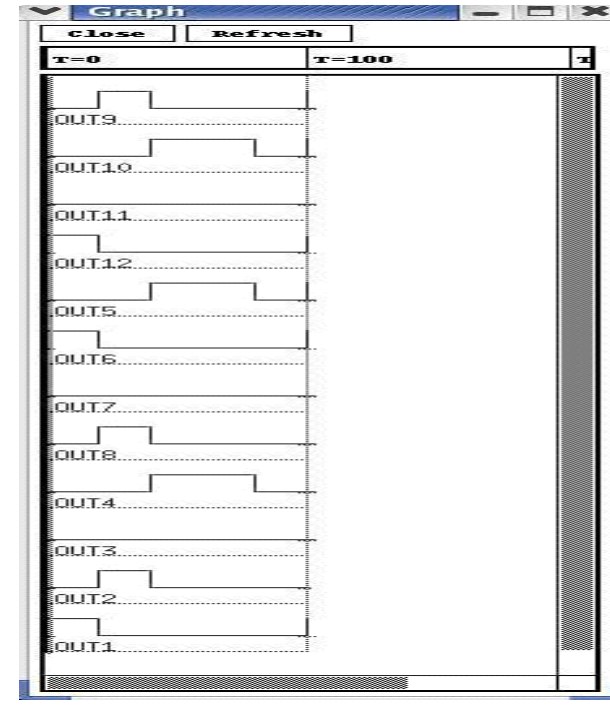

Fig.7: The output of the XHATCH simulator.

\section{Concluding Remarks}

An innovative design of a solely optical architecture implementing the centralized wavelength conversion module of the CWC protocol is presented. The use of the Optically Controlled Wavelength Conversion Circuit instead of an electronic control circuit leads to a significant improvement in the performance of the system. Moreover, since the network hub represents a single point of failure, the use of the optical wavelength conversion module instead of the 
electronic one leads to an increase in the system's reliability.

\section{References}

[1] G.I. Papadimitriou, and A.S. Pomportsis, "WavelengthConversion-Based Protocols for Single-Hop Photonic Networks with Bursty Traffic", Phot. Network Communications. 1:4 (1999) 263-271.

[2] G.I. Papadimitriou, P.A. Tsimoulas, M.S. Obaidat, and A. S. Pomportsis, Multiwavelength Optical LANs, in: John Wiley \& Sons Ltd (Ed.), West Sussex, England, 2003.

[3] A.N. Miliou, G.I. Papadimitriou, and A.S. Pomportsis, "Designing an All-Optical Packet Filtering Module for WDM Broadcast-and-Select Star Networks", Optics \& Laser Tech. 32 (2000) 317-321.
[4] R.C. Alferness, "Guided-Wave Devices for Optical Communication”, IEEE J Quantum Electron. QE-17(6) (1981) 946-959.

[5] H.F. Jordan, V.P. Heuring, and R. Feuerstein, "Optoelectronic Time-of Flight design and the Demonstration of an All-Optical, Stored Program, Digital Computer", Proc. IEEE. 82(11) (1994) 1678-1688.

[6] P.E. Jr. Green, Fiber Optic Networks, in: Prentice-Hall, Englewood Cliffs, NJ, 1993.

[7] Xhatch User's Manual, Optoelectronic Computing Systems. OCS Technical Report 95-04, University of Colorado. 